\title{
Lack of association between cortical amyloid deposition and glucose metabolism in early stage Alzheimer's disease patients
}

\author{
Daniela Ehrlich ${ }^{1}$, Andreas Dunzinger ${ }^{2}$, Gertraud Malsiner-Walli³ , Bettina Grün ${ }^{4}$, \\ Raffi Topakian ${ }^{5}$, Marina Hodolic ${ }^{6,7}$, Elmar Kainz ${ }^{1}$, Robert Pichler ${ }^{2,8,9}$ \\ ${ }^{1}$ Department of Gerontology, Kepler University Hospital, Neuromed Campus, Linz, Austria \\ 2 Institute of Nuclear Medicine, Kepler University Hospital, Neuromed Campus, Linz, Austria \\ ${ }^{3}$ Institute for Applied Statistics, Johannes Kepler University, Linz, Austria \\ ${ }^{4}$ Institute for Statistics and Mathematics, WU University of Economics and Business, Vienna, Austria \\ 5 Department of Neurology, Klinikum Wels-Grieskirchen, Wels, Austria \\ ${ }^{6}$ Nuclear Medicine Research Department, IASON, Graz, Austria \\ ${ }^{7}$ Department of Nuclear Medicine, Faculty of Medicine and Dentistry, Palacky University Olomouc, Olomouc, Czech Republic \\ 8 Institute of Nuclear Medicine, Steyr Hospital, Steyr, Austria \\ ${ }^{9}$ Department of Radiology, Clinic of Nuclear Medicine, Medical University Graz, Graz, Austria
}

Radiol Oncol 2022; 56(1): 23-31

Received 12 October 2021

Accepted 9 November 2021

Correspondence to: Prof. Marina Hodolic, M.D., Ph.D, Nuclear Medicine Research Department, IASON GmbH, Feldkirchner Straße 4, A-8054 Graz Seiersberg, Austria. Phone: + 43664830 9492; E-mail: marina.hodolic@gmail.com

Disclosure: No potential conflicts of interest were disclosed.

This is an open access article under the CC BY-NC-ND license (http://creativecommons.org/licenses/by-nc-nd/4.0/).

Background. Beta amyloid $(A \beta)$ causes synaptic dysfunction leading to neuronal death. It is still controversial if the magnitude of $A \beta$ deposition correlates with the degree of cognitive impairment. Diagnostic imaging may lead to a better understanding the role of $A \beta$ in development of cognitive deficits. The aim of the present study was to investigate if $A \beta$ deposition in the corresponding brain region of early stage Alzheimer's disease (AD) patients, directly correlates to neuronal dysfunction and cognitive impairment indicated by reduced glucose metabolism.

Patients and methods. In 30 patients with a clinical phenotype of AD and amyloid positive brain imaging, 2-[18F] fluoro-2-deoxy-d-glucose (FDG) PET/CT was performed. We extracted the average [18F] flutemetamol (Vizamyl) uptake for each of the 16 regions of interest in both hemispheres and computed the standardized uptake value ratio (SUVR) by dividing the Vimazyl intensities by the mean signal of positive and negative control regions. Data were analysed using the $R$ environment for statistical computing and graphics.

Results. Any negative correlation between $A \beta$ deposition and glucose metabolism in 32 dementia related and corresponding brain regions in AD patients was not found. None of the correlation coefficient values were statistically significant different from zero based on two-sided $\mathrm{p}$ - value.

Conclusions. Regional A deposition did not correlate negatively with local glucose metabolism in early stage AD patients. Our findings support the role of $A \beta$ as a valid biomarker, but does not permit to conclude that $A \beta$ is a direct cause for an aberrant brain glucose metabolism and neuronal dysfunction.

Key words: Alzheimer disease, PET, tau, FDG

\section{Introduction}

Alzheimer's disease (AD) is the most common cause of dementia in the elderly people. The hall- mark pathologies include beta-amyloid $(\mathrm{A} \beta)$ depositions in plaques and brain vessels, tau pathology, microglia activation, and inflammation. The causes of $\mathrm{AD}$ are unknown; however, $\mathrm{A} \beta$ may play an im- 
portant role in development of AD. According to the amyloid cascade hypothesis, $A \beta$ causes synaptic dysfunction and neuronal death leading to cognitive impairment. ${ }^{1,2}$

However, several studies suggested only a modest correlation between $A \beta$ pathology and cognition. There is little correlation between amyloid plaques location at autopsy and affected brain regions according to patient's clinical symptoms. ${ }^{3} \mathrm{~A}$ large burden of amyloid plaques is found in subjects without cognitive deficits. ${ }^{4}$ Amyloid plaque formation does occur late in hippocampus, although this structure is the first to fail clinically. ${ }^{5}$ Indeed, the reduction of plaque load in the brain in therapeutic trials has not yielded to cognitive benefit in AD patients. ${ }^{6}$

In contrast, the burden of neurofibrillary tangles, consisting of hyperphosphorylated tau, correlates with the degree of cognitive impairment in AD. ${ }^{7}$ In vivo studies using PET tracers for tau replicated these findings showing that more advanced Braak stages are associated with decreased global cognitive status. ${ }^{8,9}$ Tau pathology leads to a reduced glucose metabolism correlating with cognitive decline. ${ }^{10}$ Thus, tau pathology rather than amyloid accumulation, may contribute to cognitive dysfunction in AD patients.

Imaging may lead to a better understanding the role of $A \beta$ in the development of cognitive deficits. Techniques such as 2-[18F]fluoro-2-deoxy-d-glucose (FDG) Positron Emission Tomography (PET) or amyloid PET are widely used for supporting the diagnosis of dementia. To exclude cerebral pathologies, such as tumors, subdural hematoma or normal pressure hydrocephalus, magnetic resonance imaging (MRI) is used. Morphologically, MRI may detect a hippocampal volume reduction in $\mathrm{AD}$ [11]. Amyloid PET allows in vivo detection of amyloid plaques indicating the pathophysiologic state of dementia. Several tracers, such as $18[\mathrm{~F}]$ florbetapir, 18[F] flutemetamol and 18[F] florteban are approved by the US Food and Drug Administration (FDA). Timing of amyloid accumulation is at a preclinical stage of AD. ${ }^{12}$

However, healthy individuals without cognitive symptoms can have a positive amyloid PET scan. Thus, amyloid imaging may be helpful for differential diagnosis in early onset dementia, particularly to rule out AD dementia. FDG PET is an important tool to detect early neurodegenerative dementia, differentiate neurodegenerative dementia or comorbidity of other neurodegenerative disease. FDG PET is described as a neuronal injury biomarker in $\mathrm{AD}$ and neuronal dysfunction is indicated by reduced glucose metabolism. ${ }^{13}$ In AD patients, FDG PET demonstrates a glucose metabolic reduction in the parietotemporal association cortices, posterior cingulate and precuneus regions. ${ }^{14}$ In the later stages of $\mathrm{AD}$, hypometabolic regions spread to the frontal association cortices. ${ }^{15}$ In patients with mild cognitive impairment (MCI), the transitional stage between aging and AD, FDG PET appears to add the greatest prognostic information. ${ }^{16}$

According to the amyloid cascade hypothesis, in brain regions with amyloid deposition, neuronal injury and an altered FDG metabolism may be expected. However, several studies showed controversial findings regarding the correlation of amyloid deposition and glucose metabolism. ${ }^{17-19}$ Thus, it is still not clear if amyloid deposition comparably to tau pathology is a leading cause of cognitive impairment.

In this study, we included patients with a clinical phenotype of $\mathrm{AD}$ with cognitive dysfunction and amyloid deposition in cortical brain areas as detected by $\mathrm{A} \beta$ binding PET tracer.

The aim was to investigate if $A \beta$ deposition in the corresponding brain region directly correlates to neuronal dysfunction indicated by reduced glucose metabolism. Such a correlation should be easy and convenient to recognize when clinically AD is at a relatively early stage and impaired glucose metabolism is still restricted to certain areas and not globalized. Therefore, our cohort consists mostly of patients at the early stage of disease.

\section{Patients and methods}

\section{Patients}

Ninety patients underwent amyloid PET analysis at the Institute of Nuclear Medicine, Kepler University Hospital, Neuromed Campus, Linz, between January 2016 and November 2017. These patients were assigned from Departments of Gerontology, Neurology or Psychiatry of various hospital institutions located in upper Austria. At least all amyloid positive patients $(n=30)$ underwent a comprehensive clinical and neuropsychological evaluation. Probable AD was diagnosed clinically and according to the S3 guidelines for dementia by experienced clinicians. ${ }^{20}$ For screening, the mini mental state examination (MMSE) according to Folstein was performed. ${ }^{21}$ Each subject underwent computed tomography or MRI to rule out any structural abnormalities, such as brain tumours, hematomas, hydrocephalus or ischemia, 
as the cause for dementia. Vitamin deficiencies and thyroid abnormalities were excluded by blood analysis. FDG PET, amyloid PET and neuropsychological testing were acquired within 60 days. Brain $\mathrm{A} \beta$ deposition was quantified by performing PET scans using the tracer 18[F] flutemetamolm (Vizamyl). In a standardized procedure patients were rated as amyloid positive $(n=30$, age $65.0 \pm$ 14.3 years) or amyloid negative $(n=60$, age $64.6 \pm$ 8.7 years).

FDG PET was used to evaluate brain glucose metabolism. It was rated in clinical routine by experienced clinicians blinded to clinical symptoms using the Neuro Q, Version 3.5, 2007-analysis system, a schematic summary, comparing the patients scan to the scan of an asymptomatic control group. For our investigation, we selected 32 dementia related brain regions as regions of interest (Table 1). The regions of interest were based on brain areas, which are suggested by the Neuro Q program and include all dementia related brain regions. For each region NeuroQ compares the FDG metabolism to that of a cohort of normal persons, numbers indicate extent of standard deviation in comparison to a normal situation. Negative numbers represent relative hypometabolism.

For correlation, we manually defined cortical areas of representative gyri in dementia related brain regions of amyloid positive PET scans and excluded non cortical areas and sulci to avoid bias. Pons and the cerebellum represent the reference regions for positive and negative control within each brain. Further, we evaluated the Vizamyl uptake value ratio in corresponding brain regions. We extracted the average Vizamyl uptake for each of the 16 regions of interest in both hemispheres and computed the standardized uptake value ratio (SUVR) by dividing the Vimazyl intensities by the mean signal of the individual positive and negative control regions as mentioned above.

\section{Positron emission tomography}

All PET scans were obtained with a Philips Gemini GXL PET/CT. For amyloid imaging patients received $185 \mathrm{MBq}$ of 18F-Flutemetamol i.v. The tracer was distributed by GE healthcare Austria. PET/CT images were obtained $60 \mathrm{~min}$ after tracer injection.

FDG PET had been scheduled on a different day. Patients fasted for a minimum of $6 \mathrm{~h}$ before FDG injection to ensure standardized metabolic conditions. Blood glucose level was measured and had to be $<160 \mathrm{mg} \%$ in all patients. $185 \mathrm{MBq}$ of
TABLE 1. Regions of interest

superior frontal cortex
middle frontal cortex
Inferior frontal cortex
anterior cingulate cortex
posterior cingulate cortex
sensorimotoric cortex
superior lateral temporal cortex
medial anterior temporal cortex
medial posterior temporal cortex
inferior lateral anterior temporal cortex
inferior lateral posterior temporal cortex
superior parietal cortex
inferior parietal cortex
parietotemporal cortex
primary visual cortex
associative visual cortex

FDG was injected i.v.. PET images were acquired 30 min post injection (3D acquisition). The scanner acquires transaxial planes, simultaneously covering an $18 \mathrm{~cm}$ axial field of view. Eliminating the sub-sampling required in conventional techniques, line-of-response (LOR) removes averaging and consequent image degradation. Detector material is gadolinium oxyorthosilicate (GSO) with a crystal size of $4 \times 6 \times 30 \mathrm{~mm}$. A 6-slice helical CT - Philips brilliance air 6 - was used for attenuation correction. For further evaluation, data were transferred to a Hermes Medical Solutions, Sweden work station (HERMES).

PET interpretation was done visually by two experienced nuclear medicine specialists in knowledge of clinical data of the patient and consensually. The procedure and technical data are described in detail by Pichler et al. ${ }^{22,23}$

\section{Statistics}

Data were analysed using the $\mathrm{R}$ environment for statistical computing and graphics. ${ }^{24}$ Scatter plots of the amyloid mean score versus the FDG mean score visualize separately for each region and side the association. To test for association between these two scores, the Pearson correlation coefficient was determined for each region and side. Twosided p-values were calculated assuming normally distributed data. P-values were for each side corrected for multiple testing using Holm's method. ${ }^{25}$ 
(A)

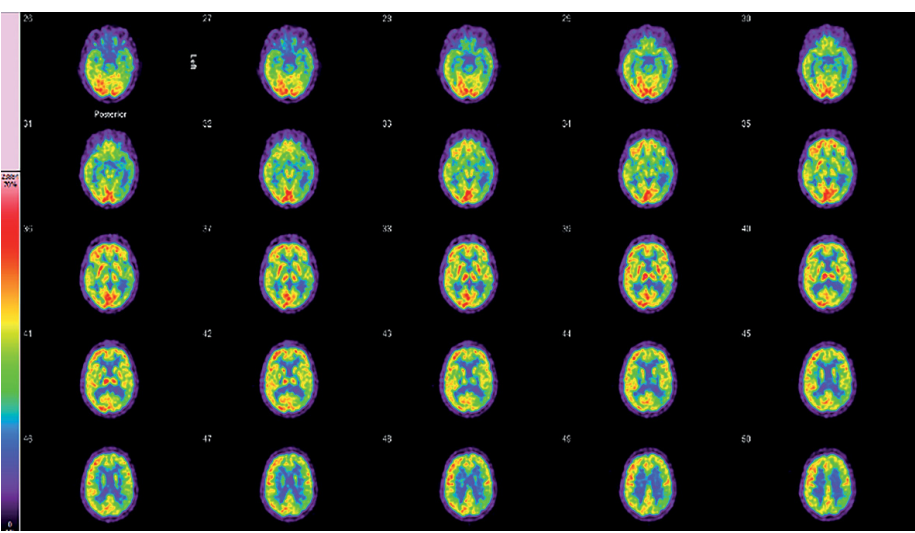

(B)

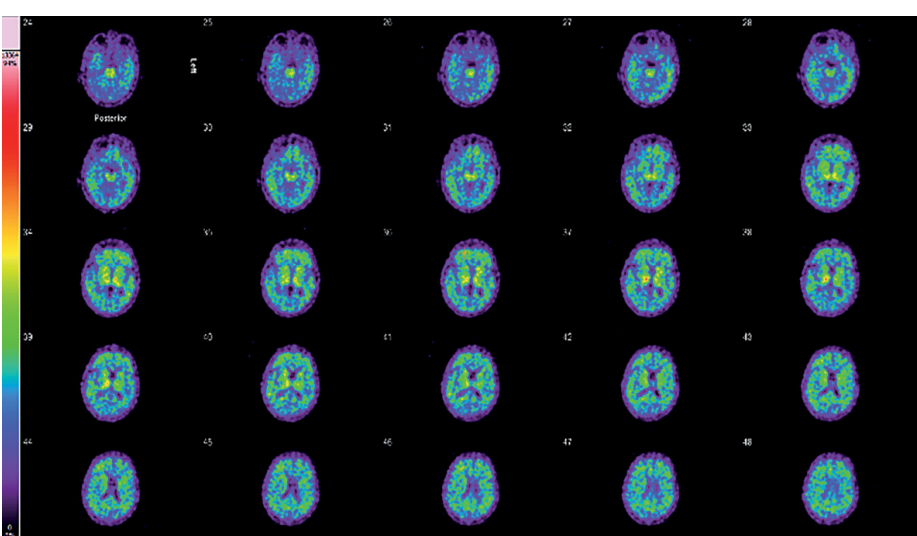

FIGURE 1. 2-[18F] fluoro-2-deoxy-d-glucose (FDG) and amyloid brain PET/CT of 59-year-old woman. (A) FDG brain PET/CT of a 59-year-old woman with a history of fluctuating cognitive impairment (mini mental state examination [MMSE] = 14/30). Glucose hypometabolism was demonstrated in the parietal dorsolateral and temporolateral, and occipatal cortical areas. The glucose metabolism in the left temporomesial area is weak. The other cortical structures show a slight attenuation of FDG metabolism. Basal ganglia show more intense uptake compared to the cortical areas. This FDG brain PET study shows the typical picture of abnormal glucose metabolism that occurs in Alzheimer's disease (AD) and is additionally compatible with pronounced microvascular changes. (B) On the amyloid PET a non-specific tracer accumulation from the pons to the basal ganglia is evident. PET images of the white matter demonstrate individual non-specific enrichments. In the frontal and temporal cortices as well as sporadically in the parietal cortical areas, a pathological tracer accumulation occurs. This global cortical tracer uptake is consistent with the neuropathology of AD.

The study was done in accord with ethical standards and in accord with the Helsinki Declaration of 1975.

\section{Results}

In clinical routine, 90 amyloid PET scans for diagnosis of dementia were performed. In detail, AD was diagnosed in 30 patients ( $65.0 \pm 14.3$ years), 16 male and 14 female. All patients with amyloid pos- itive PET scan underwent also PET scanning with FDG and computed tomography (CT) or MRI, as well as neuropsychological and clinical examination.

In the $\mathrm{AD}$ group mean score MMSE was $23 \pm 5$ $(n=30)$.

Figures 1 and 2 demonstrate FDG PET and amyloid positive PET images of patients with the clinical suspected diagnosis of AD.

In 43 subjects with amyloid negative PET scans a MMSE was performed. In the non AD group the mean score MMSE was $26 \pm 3(\mathrm{n}=43)$. Subjects with negative amyloid PET ( $\mathrm{n}=60,24$ female, 36 male, age $64.6 \pm 8.7$ ) received the following diagnosis according to ICD-10: affective disorders $(\mathrm{n}=$ 33), Parkinson's disease $(n=3)$, psychoorganic syndrom (POS, $n=3$ ), Hashimoto's encephalopathy ( $n$ $=1)$, hepatic encephalopathy $(n=1)$, frontotemporal dementia (FTD, $\mathrm{n}=8$ ), vascular dementia (vaD, $\mathrm{n}=8)$ and $\beta$-amyloid associated angiopathy $(\mathrm{n}=3)$.

As shown in Figure (3) and Figure (4) we did not find any significant negative correlation between amyloid deposition and glucose metabolism in 32 dementia related and corresponding brain regions in $\mathrm{AD}$ patients. The estimated correlation coefficient values differed between 0.48 and -0.32 . None of the correlation coefficient values were statistically significant different from zero based on two-sided p- value at significance level 0.05 after correcting for multiple testing for each side using Holm's method. No statistical evidence was found to confirm a negative correlation between amyloid deposition and glucose metabolism in general or specifically for some brain regions.

\section{Discussion}

There is an ongoing debate to which extent amyloid is related to AD pathology. The concept of a direct mechanism leading to clinical manifestation lead to various trials of vaccination therapies. As therapeutic success was disappointing the pathophysiological role of amyloid in AD had to be rediscussed.

In AD pathology, the amyloid cascade hypothesis may play a fundamental role. ${ }^{2}$ Plaques in AD brains consist of insoluble $A \beta$ peptides cleaved by different secretases from the amyloid precursor protein (APP). ${ }^{26}$ The cleavage results in $\mathrm{A} \beta-40$ with a length of 40 amino acids and $A \beta-42$ with a length of 42 amino acids, which is the plaque proning form. Distinct plaque subtypes with low (diffuse plaques) and high (cored or neurotic plaques) 
proportion of fibrillar components have been identified. ${ }^{27}$

In fact, insoluble $A \beta$ exceeds soluble forms of $\mathrm{A} \beta$ by a factor of about 100 -fold in $\mathrm{AD}$ brain. $^{28}$ However, $\mathrm{A} \beta$ does not correlate well with clinical symptoms and anti-amyloid pharmaceuticals have failed to improve significantly patient's symptoms ${ }^{3,6}$, even when amyloid deposits are efficiently reduced. ${ }^{29}$ As a possible exception, the updated analysis of the EMERGE trial showed a significant reduction in decline of global functions for the patients treated with a high dose of aducanumab. ${ }^{30}$

Thus, it is still not clear if $\mathrm{A} \beta$ directly leads to neuronal dysfunction. High levels of $A \beta$ may subsequently lead to a downstream of pathological events, including tau pathology, inflammation, oxidative stress, excitotoxicity, loss of synaptic connections, and cell death, causing the clinical symptoms of AD. Levels of prefibrillar A $\beta$ forms, such as soluble oligomers and protofibrils, correlate better than plaques with disease severity. ${ }^{31}$ This may indicate that soluble species are the neurotoxic form of $A \beta$ leading to neurodegeneration. ${ }^{31}$

$\mathrm{A} \beta$ deposition is a valid biomarker to support AD diagnostic. ${ }^{32}$ Available PET radioligands visualizing $A \beta$ bind to insoluble fibrils, such as $\mathrm{A} \beta$ plaques. Recently, several 18F-labeled tracers were designed including flobetapir ([18F]AV-45), flutemetamol ([18F]GE067), florbetaben ([18F] BAY94-9172) [28, 33-36]. We used [18F]flutemetamol PET as a surrogate marker for brain amyloid deposition. Several studies suggested a high correlation between $[18 \mathrm{~F}]$ flutemetamol retention and neuropathologic findings. ${ }^{37-40}$ However, amyloid specific tracers may not be able to provide an accurate measurement of $A \beta$. In fact, there is a lack of data of in vivo A $\beta$ specificity. ${ }^{41}$ Amyloid PET scan is able to rule out an AD diagnosis. Amyloid PET may have an additive, but primarily confirmatory role as a diagnostic marker in patients suspected of early-onset AD. An amyloid-positive PET scan often supports or changes diagnosis into AD. ${ }^{42}$ Amyloid pathology is also present in other forms of dementia and interpreted as mixed or copathology and not always the primary cause of the clinical manifestation of dementia.

FDG PET, a neuroimaging tool in AD, plays an important role in discriminating different forms of dementia. ${ }^{12,34}$ Decreased glucose metabolism in temporal and parietal cortex indicates synaptic dysfunction and in contrast to amyloid deposition, this occurs mainly in the symptomatic phase of AD. ${ }^{28}$ In the present study, we investigated a possible correlation between local amyloid deposition

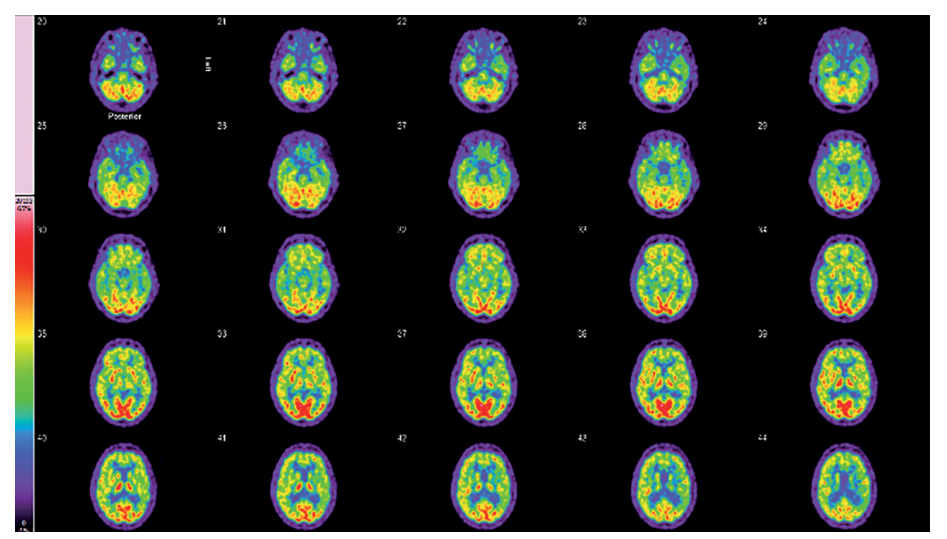

(A)

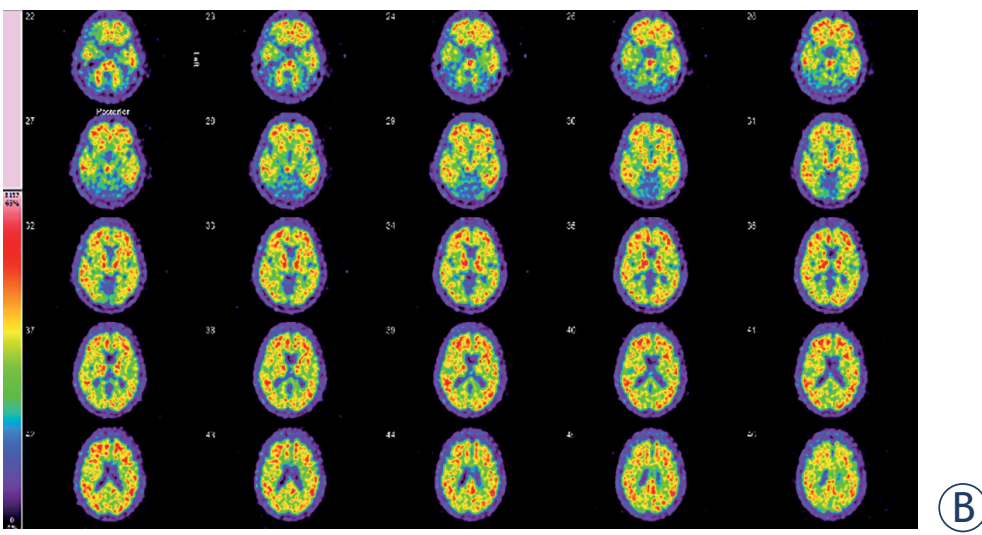

FIGURE 2. FDG and amyloid brain PET/CT of a 70-year-old woman. (A) 2-[18F] fluoro2-deoxy-d-glucose (FDG) brain PET/CT of a 70-year-old woman, who presented with a history of cognitive decline (mini mental state examination [MMSE] = 15/30). The glucose metabolism in the cerebral cortex is inhomogenous and moderately attenuated. In the cerebellum, normal glucose metabolism was demonstrated. This FDG brain PET study does not show the typical picture of abnormal glucose metabolism that occurs in Alzheimer's disease (AD), but temporomesial and temporolateral some decreased tracer uptake can be observed. Additionally the images are compatible with pronounced microvascular changes. (B) Amyloid PET images demonstrate pathologically increased tracer accumulation in the entire brain, more pronounced in the frontal and temporal cortical areas. This is compatible with the diagnosis of AD.

and glucose metabolism in dementia related corresponding brain regions in AD patients. In an early stage of AD impaired glucose metabolism is still restricted to certain areas and not globalized. ${ }^{43}$ Our cohort consists mostly of patients at the early stage of disease, which is a result of reasonable referrals. Clinical impact for different diagnosis of dementia in an advanced state of disease is questionable, because all therapeutical strategies are more helpful at an early phase of the disease. The availability of both PET modalities in 30 patients diagnosed for $\mathrm{AD}$ in the present study allowed investigating the correlation of amyloid deposition and glucose metabolism, retrospectively. 

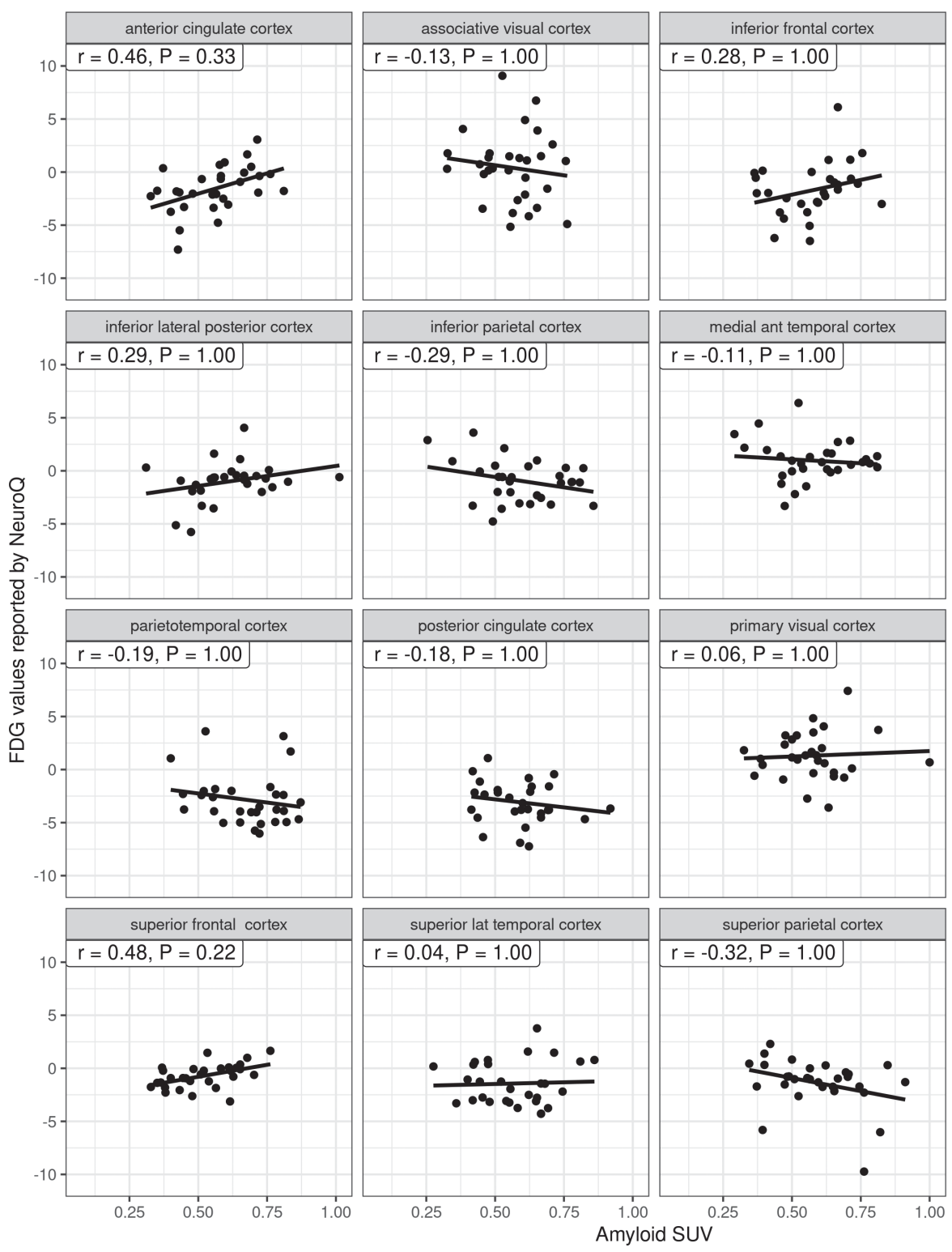
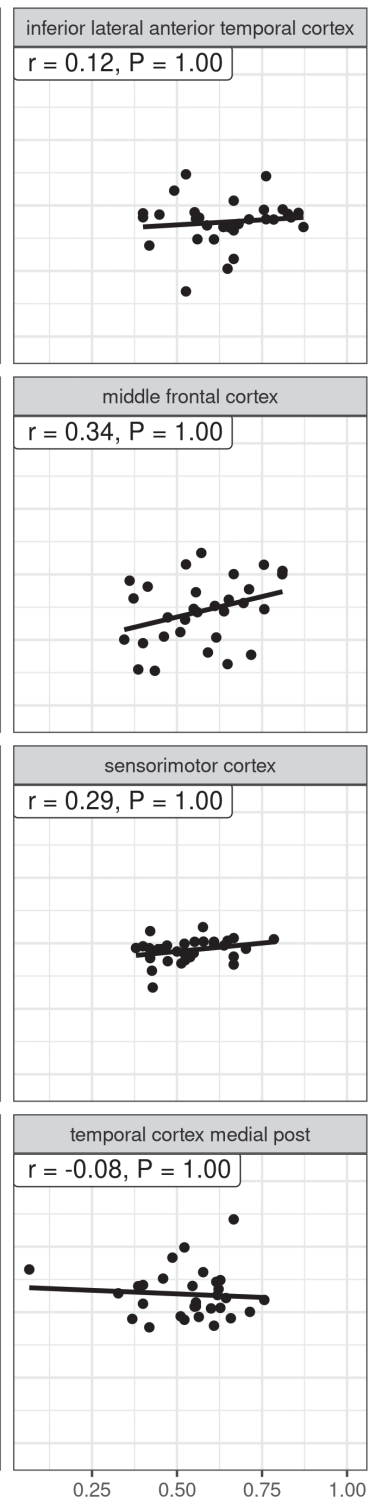

FIGURE 3. Scatter plots of the amyloid standardized uptake value (SUV) (on the x-axis) and the 2-[18F] fluoro-2-deoxy-d-glucose (FDG) values represented by NeuroQ (on the y-axis) for the 30 amyloid positive patient for the left side. Each panel represents a brain regions with the name indicated in the strip. Least-squares regression lines with slope proportional to the Pearson correlation coefficient indicate the association. The Pearson correlation ( $r$ ) and the associated p-value (P) are shown in the label on the top of each panel.

To avoid bias due to computer assisted measurement we manually designated the cortical areas of dementia related brain regions and evaluated the SUVR of [18F]flutemetamol. Compared to previous investigations ${ }^{17-19}$ we included a higher number of AD patients. Engler et al. found a negative correlation with metabolism in parietal cortex in $16 \mathrm{AD}$ patients. ${ }^{17}$ Another author showed that a higher amyloid tracer uptake correlated with lower regional glucose metabolism in $19 \mathrm{AD}$ pa- tients. ${ }^{18}$ We did not find any negative correlation of amyloid tracer uptake and glucose metabolism in corresponding brain areas in $30 \mathrm{AD}$ patients. Our data supports the concept that amyloid depositions may not be the direct cause of dysfunctional metabolism.

One limitation of this study is that measurement of $A \beta 40, A \beta 42$ and phosphorylated tau in the cerebrospinal fluid (CSF) was not performed ${ }^{44}$ and thus, the correlation between CSF amyloid levels 

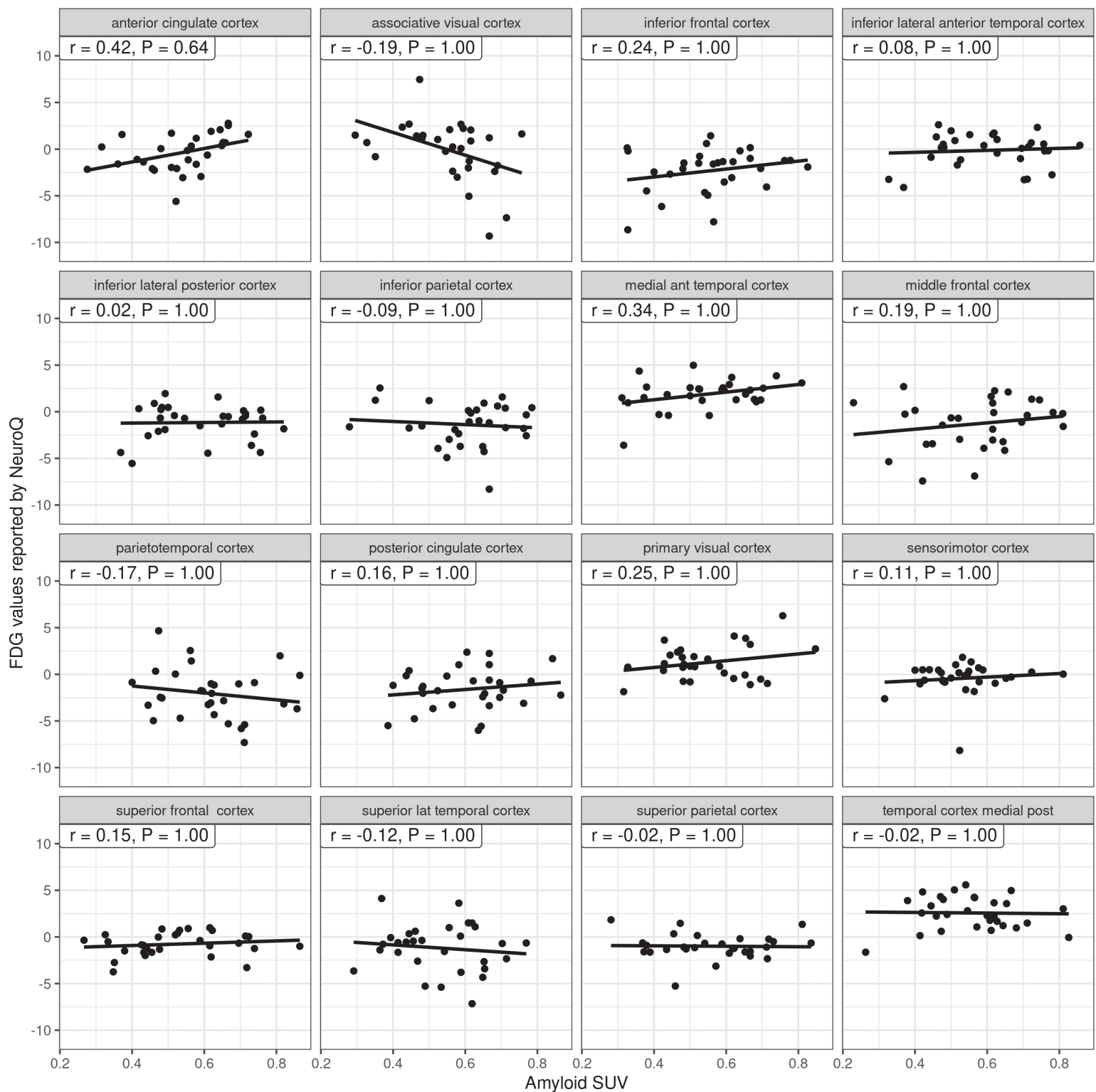

FIGURE 4. Scatter plots of the amyloid standardized uptake value (SUV) (on the x-axis) and the 2-[18F] fluoro-2-deoxy-d-glucose (FDG) values represented by NeuroQ (on the $y$-axis) for the 30 amyloid positive patient for the right side. Each panel represents a brain regions with the name indicated in the strip. Least-squares regression lines with slope proportional to the Pearson correlation coefficient indicate the association. The Pearson correlation ( $r$ ) and the associated $p$-value (P) are shown in the label on the top of each panel.

and amyloid tracer uptake could not be shown. If $\mathrm{A} \beta$ deposition plays a role in the development of cognitive deficits in $\mathrm{AD}$, the lack of direct correlations requires other involved mechanisms such as tau pathology. $2,45,46$

The hyperphosphorylation and abnormal aggregation of tau, a microtubule-associated protein essential to neuronal stability and functioning, is a hallmark in AD pathology. Tau imaging revealed that neurofibrillary tangels are mainly located in the hippocampus and associative cortical regions. ${ }^{47}$ Tau PET imaging may serve as a valuable and early biomarker for the localization of neuronal injury. In contrast to $\mathrm{A} \beta$ accumulation, tau may cause cognitive decline mediated by glucose hypometabolism. Indeed, tau pathology was observed in brain regions related to clinical symptoms and overlapped with areas of hypometabolism. ${ }^{48.49}$ Exactly what we were not able to show for the relation of amyloid and glucose metabolism. $A \beta$ may play an indirect 
role in AD pathology in the development of NFTs. It is likely, that $\mathrm{A} \beta$ indirectly promotes tau phosphorylation through upregulation of kinases such as GSK-3 $\beta$ and CDK5, which phosphorylate tau..$^{50}$

\section{Conclusios}

The focus of the study was to investigate the correlation between local amyloid deposition and glucose metabolism in vivo at corresponding brain areas. Therefore, we manually designated the areas of interest in an early stage of disease, which in that manner has not been performed before. We showed that regional amyloid deposition did not correlate negatively with local glucose metabolism. Our findings support the role of $A \beta$ as a valid biomarker, but does not permit to conclude that $A \beta$ is a direct cause for an aberrant glucose metabolism and neuronal dysfunction. On the contrary our data added a piece of puzzle to the concept that amyloid does not directly cause AD pathology but has to be considered as a prerequisite only.

\section{Acknowledgements}

The authors are grateful to Ms. Silke Kern and Ms. Sieglinde Prechtl and to their whole team of technicians for invaluable support at the Institute of Nuclear Medicine.

Contribution of authors: DE, MH \& RP manuscript writing; AD \& RP nuclear imaging; GMW \& BG statistics; DE, RT \& EK clinical part.

\section{References}

1. Hardy JA, Higgins GA. Alzheimer's disease: the amyloid cascade hypothesis Science 1992; 256: 184-5. doi: 10.1126/science. 1566067

2. Hardy J, Selkoe DJ. The amyloid hypothesis of Alzheimer's disease: progress and problems on the road to therapeutics. Science 2002; 297: 353-6. doi: 10.1126/science.1072994

3. Murphy MP, LeVine H. Alzheimer's disease and the amyloid-beta peptide. $J$ Alzheimers Dis 2010; 19: 311-23. doi: 10.3233/JAD-2010-1221

4. Jack CR Jr, Wiste HJ, Knopman DS, Vemuri $P$, Mielke MM, Weigand $\mathrm{SD}$, et al. Rates of beta-amyloid accumulation are independent of hippocampal neurodegeneration. Neurology 2014; 82: 1605-12. doi: 10.1212/ WNL.0000000000000386

5. Altmann A, Ng B, Landau SM, Jagust WJ, Greicius MD. Regional brain hy pometabolism is unrelated to regional amyloid plaque burden. Brain 2015; 138: 3734-46. doi: 10.1093/brain/awv278

6. Salloway S, Sperling R, Fox NC, Blennow K, Klunk W, Raskind M, et al Bapineuzumab 301 and 302 Clinical Trial Investigators. Two phase 3 trials of bapineuzumab in mild-to-moderate Alzheimer's disease. $N$ Engl J Med 2014; 370: 322-33. doi: 10.1056/NEJMoa1304839
7. Nelson PT, Alafuzoff I, Bigio EH, Bouras C, Braak H, Cairns NJ, et al. Correlation of Alzheimer disease neuropathologic changes with cognitive status: a review of the literature. J Neuropathol Exp Neurol 2012; 71: 36281. doi: 10.1097/NEN.0b013e31825018f7

8. Cho H, Choi JY, Hwang MS, Lee JH, Kim YJ, Lee HM, et al. Tau PET in Alzheimer disease and mild cognitive impairment. Neurology 2016; 87: 375-83. doi: 10.1212/WNL.0000000000002892

9. Schöll M, Ossenkoppele R, Strandberg O, Palmqvist S, Jögi J, Ohlsson T, et al. Distinct 18F-AV-1451 tau PET retention patterns in early- and late-onset Alzheimer's disease. Brain 2017; 140: 2286-94. doi: 10.1093/brain/awx171

10. Saint-Aubert L, Almkvist O, Chiotis K, Almeida R, Wall A, Nordberg A. Regional tau deposition measured by [18F]THK5317 positron emission tomography is associated to cognition via glucose metabolism in Alzheimer's disease. Alzheimers Res Ther 2016; 8: 38. doi: 10.1186/s13195-016-0204-z

11. Jack CR Jr, Petersen RC, Xu YC, O'Brien PC, Smith GE, Ivnik RJ, et al. Prediction of $A D$ with MRI-based hippocampal volume in mild cognitive impairment. Neurology 1999; 52: 1397-403.

12. Villemagne VL, Burnham $S$, Bourgeat $P$, Brown $B$, Ellis KA, Salvado $O$, et al. Australian Imaging Biomarkers and Lifestyle (AIBL) Research Group. Amyloid $\beta$ deposition, neurodegeneration, and cognitive decline in sporadic Alzheimer's disease: a prospective cohort study. Lancet Neurol 2013; 12: 357-67. doi: 10.1016/S1474-4422(13)70044-9

13. McKhann GM, Knopman DS, Chertkow H, Hyman BT, Jack CR Jr, Kawas $\mathrm{CH}$, et al. The diagnosis of dementia due to Alzheimer's disease: recommendations from the National Institute on Aging-Alzheimer's Association workgroups on diagnostic guidelines for Alzheimer's disease. Alzheimers Dement 2011; 7: 263-9. doi: 10.1016/j.jalz.2011.03.005

14. Shivamurthy VK, Tahari AK, Marcus C, Subramaniam RM. Brain FDG PET and the diagnosis of dementia. AJR Am J Roentgenol 2015; 204: W76-85. doi: 10.2214/AJR.13.12363

15. Ishii K. PET approaches for diagnosis of dementia. AJNR Am J Neuroradiol 2014; 35: 2030-8. doi: 10.3174/ajnr.A3695

16. Shaffer JL, Petrella JR, Sheldon FC, Choudhury KR, Calhoun VD, Coleman $\mathrm{RE}$, et al. Predicting cognitive decline in subjects at risk for Alzheimer disease by using combined. Radiology 2013; 266: 583-91. doi: 10.1148/ radiol.12120010

17. Engler H, Forsberg A, Almkvist O, Blomquist G, Larsson E, Savitcheva I, et al. Two-year follow-up of amyloid deposition in patients with Alzheimer's disease. Brain 2006; 129: 2856-66. doi: 10.1093/brain/awl178

18. Edison P, Archer HA, Hinz R, Hammers A, Pavese N, Tai YF, et al. Amyloid, hypometabolism, and cognition in Alzheimer disease: An [11C]PIB and [18F]FDG PET study. Neurology 2007; 68: 501-8. doi: 10.1212/01. wnl.0000244749.20056.d4

19. Cohen AD, Price JC, Weissfeld LA, James J, Rosario BL, Bi W, et al. Basal cerebral metabolism may modulate the cognitive effects of $A \beta$ in mild cognitive impairment: an example of brain reserve. J Neurosci 2009; 29: 14770-8. doi: 10.1523/JNEUROSCI.3669-09.2009

20. Deuschl G, Maier W, et al; Steuerungsgruppe. S3- Guideline dementias. [German]. In: German Society for Neurology, editor. Guidelines for diagnostics and therapy in neurology. [German]. 2016. [cited 2021 Sep 12]. Available at: www.dgn.org/leitlinien

21. Folstein MF, Folsten SE, McHugh PR. Mini-mental state: a practical method for grading the cognitive state of patients for the clinician. J Psychiatr Res 1975; 12: 189-98. doi: 10.1016/0022-3956(75)90026-6

22. Pichler R, Dunzinger A, Wurm G, Pichler J, Weis $S$, Nussbaumer $K$, et al. Is there a place for FET PET in the initial evaluation of brain lesions with unknown significance? Eur J Nucl Med Mol Imaging 2010; 37: 1521-8. doi: 10.1007/s00259-010-1457-6

23. Hodolic M, Topakian R, Pichler R. ${ }^{18} \mathrm{~F}$-fluorodeoxyglucose and ${ }^{18} \mathrm{~F}$-flumazenil positron emission tomography in patients with refractory epilepsy. Radiol Oncol 2016; 50: 247-53. doi: 10.1515/raon-2016-0032

24. R Core Team. A language and environment for statistical computing. Vienna Austria: R Foundation for Statistical Computing; 2019. [cited 2021 Sep 13]. Available at: http://www.R-project.org/

25. Holm S. A simple sequentially rejective multiple test procedure. Scand J of Stat 1979; 6: 65-70.

26. Murphy MP, LeVine $\mathrm{H}$. Alzheimer's disease and the amyloid-beta peptide. J Alzheimers Dis 2010; 19: 311-23. doi: 10.3233/JAD-2010-1221 
27. Dickson TC, Vickers JC. The morphological phenotype of beta-amyloid plaques and associated neuritic changes in Alzheimer's disease. Neuroscience 2001; 105: 99-107. doi: 10.1016/s0306-4522(01)00169-5

28. Vlassenko AG, Benzinger TL, Morris JC. PET amyloid-beta imaging in preclinical Alzheimer's disease. Biochim Biophys Acta 12012; 1822: 370-9. doi: 10.1016/j.bbadis.2011.11.005

29. Lowe SL, Willis BA, Hawdon A, Natanegara F, Chua L, Foster J, et al. Donanemab (LY3002813) dose-escalation study in Alzheimer's disease. Alzheimers Dement (N Y) 7: e12112. doi: 10.1002/trc2.12112

30. Huang LK, Chao SP, Hu CJ. Clinical trials of new drugs for Alzheimer disease. J Biomed Sci 2020; 27: 18. doi: 10.1186/s12929-019-0609-7

31. Tomic JL, Pensalfini A, Head E, Glabe CG. Soluble fibrillar oligomer levels are elevated in Alzheimer's disease brain and correlate with cognitive dysfunction. Neurobiol Dis 2009; 35: 352-8. doi: 10.1016/j.nbd.2009.05.024

32. Weiner MW, Veitch DP, Aisen PS, Beckett LA, Cairns NJ, Cedarbaum J, et al. 2014 Update of the Alzheimer's Disease Neuroimaging Initiative: a review of papers published since its inception. Alzheimers Dement 2015; 11: e1-120. doi: 10.1016/j.jalz.2014.11.001

33. Small GW, Kepe V, Ercoli LM, Siddarth P, Bookheimer SY, Miller KJ, et al. PET of brain amyloid and tau in mild cognitive impairment. N Engl J Med 2006; 355: 2652-63. doi: 10.1056/NEJMoa054625

34. Rowe CC, Ackerman U, Browne W, Mulligan R, Pike KL, O'Keefe G, et al. Imaging of amyloid beta in Alzheimer's disease with 18F-BAY94-9172, a novel PET tracer: proof of mechanism. Lancet Neurol 2008; 7: 129-35. doi: 10.1016/S1474-4422(08)70001-2

35. Koole M, Lewis DM, Buckley C, Nelissen N, Vandenbulcke M, Brooks DJ, et al. Whole-body biodistribution and radiation dosimetry of 18F-GE067: a radioligand for in vivo brain amyloid imaging. J Nucl Med 2009; 50: 818-22. doi: 10.2967/jnumed.108.060756

36. Kung HF, Choi SR, Qu W, Zhang W, Skovronsky D. 18F stilbenes and styrylpyridines for PET imaging of a beta plaques in Alzheimer's disease: a miniperspective. J Med Chem 2010; 53: 933-41. doi: 10.1021/jm901039z

37. Frings L, Hellwig S, Spehl TS, Bormann T, Buchert R, Vach $W$, et al. Asymmetries of amyloid- $\beta$ burden and neuronal dysfunction are positively correlated in Alzheimer's disease. Brain 2015; 138: 3089-99. doi: 10.1093/ brain/awv229

38. Jack CR Jr, Wiste HJ, Knopman DS, Vemuri P, Mielke MM, Weigand $\mathrm{SD}$, et al. Rates of beta-amyloid accumulation are independent of hippocampal neurodegeneration. Neurology 2014; 82: 1605-12. doi: 10.1212/ WNL.0000000000000386

39. Scahill RI, Schott JM, Stevens JM, Rossor MN, Fox NC. Mapping the evolution of regional atrophy in Alzheimer's disease: unbiased analysis of fluid-registered serial MRI. Proc Natl Acad Sci USA 2002; 99: 4703-7. doi: $10.1073 /$ pnas.052587399

40. Pearson RC, Esiri MM, Hiorns RW, Wilcock GK, Powell TP. Anatomical correlates of the distribution of the pathological changes in the neocortex in Alzheimer disease. Proc Natl Acad Sci USA 1985; 82: 4531-4. doi: 10.1073/ pnas.82.13.4531

41. Kepe V, Moghbel MC, Långström B, Zaidi H, Vinters HV, Huang SC, et al. Amyloid- $\beta$ positron emission tomography imaging probes: a critical review. J Alzheimers Dis 2013; 36: 613-31. doi: 10.3233/JAD-130485

42. Zwan MD, Bouwman FH, Konijnenberg E, van der Flier WM, Lammmertma AA, Verhey FR, et al. Diagnostic impact of [18F]flutemetamol PET in earlyonset dementia. Alzheimers Res Ther 2017; 9: 2. doi: 10.1186/s13195-0160228-4

43. Braak $\mathrm{H}$, Braak E. Neuropathological stageing of Alzheimer-related changes. Acta Neuropathol 1991; 82: 239-59. doi: 10.1007/BF00308809

44. Morbelli S, Bauckneht M, Scheltens P. Imaging biomarkers in Alzheimer's Disease: added value in the clinical setting. Q J Nucl Med Mol Imaging 2017; 61: 360-71. doi: 10.23736/S1824-4785.17.03011-4

45. Bennett DA, Schneider JA, Wilson RS, Bienias JL, Arnold SE. Neurofibrillary tangles mediate the association of amyloid load with clinical Alzheimer disease and level of cognitive function. Arch Neurol 2004; 61: 378-84. doi: 10.1001/archneur.61.3.378

46. Shankar GM, Li S, Mehta TH, Garcia-Munoz A, Shepardson NE, Smith I, et al. Amyloid-beta protein dimers isolated directly from Alzheimer's brains impair synaptic plasticity and memory. Nat Med 2008; 14: 837-42. doi: $10.1038 / \mathrm{nm} 1782$
47. Braak H, Alafuzoff I, Arzberger T, Kretzschmanr H, Del Tredici K. Staging of Alzheimer disease-associated neurofibrillary pathology using paraffin sections and immunhistochemistry. Acta Neuropathol 2006; 112: 389-404. doi: 10.1007/s00401-006-0127-z

48. Dronse J, Fliessbach K, Bischof GN, Reutern von B, Faber J, Hammes J, et al. In vivo patterns of tau pathology, amyloid- $\beta$ burden, and neuronal dysfunction in clinical variants of Alzheimer's disease. J Alzheimers Dis 2017; 55: 465-71. doi: 10.3233/JAD-160316

49. Bejanin A, Schonhaut DR, La Joie R, Kramer JH, Baker SL, Sosa N, et al. Tau pathology and neurodegeneration contribute to cognitive impairment in Alzheimer's disease. Brain 2017; 140: 3286-300. doi: 10.1093/brain/awx243

50. Huber CM, Yee C, May T, Dhanala A, Mitchell CS. Cognitive decline in preclinical Alzheimer's disease: amyloid-beta versus tauopathy. J Alzheimers Dis 2018; 61: 265-81. doi: 10.3233/JAD-170490 